\title{
Compactness in spaces of measures
}

by

FLEMMING TOPSøE (Copenhagen)

1. Introduction. A famous result of Prohoror [8] states that if $X$ is a Polish space, and $\mathfrak{M}_{\leftarrow}^{1}(X)$ the space of probability measures on $X$ provided with the topology of weak convergence, then the relatively compact subsets of $\mathfrak{M}_{+}^{\mathbb{I}}(X)$ are precisely the tight ones. Independently of each other, P.A. Meyer and L. Schwartz remarked that tightness implies relative compactness even in the general setting of a Hausdorff space $X$ and the space of tight probability measures (private communications). The method consisted in reduction to the compact case. From counterexamples in Varadarajan [9] and Fernique [4] it is known that tightness is not a necessary condition for relative compactness to hold

It is the main aim of this paper to characterize the compact subsets of $\mathfrak{M}_{\leftarrow}(X)$ in general situations. The basic tool is Theorem 1 on extension of a content to a measure. This result is easily derived from the ideas contained in the proof of Theorem 1.2 in Kisyński's paper [7]; in spite of this we shall include a full proof.

Theorem 1 is also applied to derive some compactness results previously established in special cases by Dieudonné [2] and by Grothendieck [5].

2. Definitions and terminology. A paving is a non-empty set consisting of subsets of a given set $X$. For pavings we shall use a terminology resembling that of P. A. Meyers; we illustrate this by some examples. $\mathscr{A}$ is said to be a $(\bigcup \mathrm{f}, \cap \mathrm{c})$-paving if $\mathscr{A}$ is closed under finite unions and countable intersections; if in addition $\emptyset \in \mathscr{A}$ and $X \epsilon \mathscr{A}$ we speak of a $(\varnothing, X, \cup \mathrm{f}, \cap \mathrm{c})$-paving. A $(\cup \mathrm{c}, \mathrm{C})$-paving is the same as a $\sigma$-field. A $(\varnothing, X, \cap \mathrm{f}, \cup$ a)-paving is the same as a topology.

A paving $\mathscr{K}$ is said to be compact [semicompact] if every family [every countable family] of sets in $\mathscr{K}$, which has the finite intersection property, has a non-empty intersection.

A paving $\mathscr{F}$ is filtering to the left if $F_{1} \in \mathscr{F} \wedge F_{2} \in \mathscr{F} \Rightarrow \exists F \in \mathscr{F}$ : $F \subseteq F_{1} \cap F_{2}$. If $\mathscr{F}$ is filtering to the left and if $F_{0}=\bigcap\{F \mid F \in \mathscr{F}\}$, then we express this notationally by writing $\mathscr{F} \downarrow F_{0}$. For pavings filtering to the right we use the notation $\mathscr{F} \uparrow F_{0}$. 
A paring $\mathscr{G}$ separates points if to any pair $x_{1}, x_{2}$ of distinct points in $X$ we can find a pair $G_{1}, G_{2}$ of disjoint sets in $\mathscr{G}$ such that $x_{1} \in G_{1}$ and $x_{2} \in G_{2} . \mathscr{G}$ separates the sets in $\mathscr{K}$ if to any pair $K_{1}, K_{2}$ of disjoint sets in $\mathscr{K}$, we can find a pair $G_{1}, G_{2}$ of disjoint sets in $\mathscr{G}$ such that $K_{1} \subseteq G_{1}$ and $K_{2} \subseteq G_{2}$.

BY a set function we shall here mean a non-negative, possibly infinitevalued function defined on a paving. Let $\beta$ be a set function defined on the paving $\mathscr{A}$. In all the definitions given below we only require the defining relations to holds when they make sense. $\beta$ is monotone if $A_{1} \subseteq A_{2} \Rightarrow \beta A_{1} \leqslant \beta A_{2}$. $\beta$ is subadditive if $\beta\left(A_{1} \cup A_{2}\right) \leqslant \beta A_{1}+\beta A_{2}$ holds. $\beta$ is additive if $A_{1} \cap A_{2}=\emptyset \Rightarrow \beta\left(A_{1} \cup A_{2}\right)=\beta A_{1}+\beta A_{2} . \beta$ is modular if $\emptyset \in \mathscr{A}$, if $\beta \emptyset=0$, and if $\beta\left(A_{1} \cup A_{2}\right)+\beta\left(A_{1} \cap A_{2}\right)=\beta A_{1}+\beta A_{2}$ holds A monotone set function $\beta$ defined on $\mathscr{A}$ is $\sigma$-smooth [ $\tau$-smooth] with respect to the paving $\mathscr{K}$ if $\left(\mathscr{K}^{*} \subseteq \mathscr{K}\right) \wedge\left(\mathscr{K}^{*}\right.$ countable [arbitrary] $) \wedge$ $\wedge\left(\mathscr{K}^{*} \downarrow A_{0}\right) \wedge\left(A_{0} \in \mathscr{A}\right) \Rightarrow \beta A_{0}=\inf \left\{\beta A \mid \exists K \in \mathscr{K}^{*}: A \supseteq K\right\}$, provided the r.h.s. in this equation is finite. If $\emptyset_{\epsilon} \mathscr{A}$, and if we only require the last relation to hold when $A_{0}=\varnothing$, then we say that $\beta$ is $\sigma$-smooth at $\varnothing$ [ $\tau$-smooth at Ø] w. r.t. $\mathscr{K}$. If $\mathscr{K}=\mathscr{A}$ in the last definitions, we obtain the definitions of set functions which are $\sigma$-smooth, $\tau$-smooth, $\sigma$-smooth at $\emptyset$ or $\tau$-smooth at $\varnothing$. $\beta$ is said to be regular w.r.t. the paving $\mathscr{K}$ if $\mathscr{K} \subseteq \mathscr{A}$ and if $\beta A=\sup \{\beta K \mid K \subseteq A \wedge K \in \mathscr{K}\}$ holds. A finite $\beta$ is tight if $A_{1} \supseteq A_{2}$ $\Rightarrow \sup \left\{\beta A \mid A \subseteq A_{1} \backslash A_{2}\right\}=\beta A_{1}-\beta A_{2} . \beta$ is a content if $\mathscr{A}$ is a $(\varnothing, \cup \mathrm{f}$, $\cap$ f)-paving and if $\beta$ is finite, monotone, additive and subadditive.

A directed set is a set $D$ with a transitive and reflexive relation $\leqslant$ such that any finite subset of $D$ has a majorant. A net on $X$ is a mapping of a directed set into $X$. A net $\left(x_{\alpha}\right)_{a \in D}$ on $X$ is universal if, for every subset $A$ of $X$ it is either true that $x_{\alpha} \in A$, eventually (i.e. $\exists \alpha_{0} \forall a \geqslant \alpha_{0}: x_{\alpha} \in A$ ) or else we have $x_{\alpha} \in \mathrm{C} A$, eventually. Let $\left(x_{\alpha}\right)_{\alpha \in D}$ and $\left(x_{\alpha}\right)_{\beta \in E}$ be two nets on $X$ such that the mapping $\beta \rightarrow \alpha_{\beta}$ of $E$ into $D$ satisfies the requirement that, for any $\alpha_{0} \in D$, we have $\alpha_{\beta} \geqslant \alpha_{0}$, eventually; then $\left(x_{a_{\beta}}\right)_{\beta \in E}$ is called a subnet of $\left(x_{\alpha}\right)_{\alpha \in D}$. Every net has a universal subnet. The reader may wish to consult J. L. Kelley, General topology, for further comments on the notion of nets.

By a topological space we shall here mean a topological Hausdorff space. A net $\left(x_{\alpha}\right)_{\alpha \in D}$ on a topological space $X$ is said to be compact if every subnet has a further subnet which converges (or, equivalently, if every universal subnet of $\left(x_{a}\right)$ converges). A subset $A$ of $X$ is called net-compact if every net on $A$ has a convergent subnet (or, equivalently, if every universal net on $A$ converges). In case $X$ is a regular topological space, $A \subseteq X$ is net-compact if and only if $A$ is relatively compact (Bourbaki [1], chap. I, § 10, ex. 1).

A topological space is called a $G_{\delta}$-space if every closed set is a countable intersection of open sets.
In the main results, we shall consider an abstract set $X$ provided with two pavings $\mathscr{K}$ and $\mathscr{G}$. By $\mathscr{B}=\mathscr{B}(\mathscr{K})$ we denote the smallest $\sigma$-field containing every set $E \subseteq X$ for which $K \cap E \in \mathscr{K} \forall K \in \mathscr{K} . \mathfrak{M}_{+}(X)$ denotes the set of finite, non-negative measures defined on $\mathscr{B}(\mathscr{K}) . \mathfrak{M}_{+}(X ; \mathscr{K})$ denotes the set of measures in $\mathfrak{M}_{+}(X)$ which are regular w. r.t. $\mathscr{K}$. $\mathfrak{M}_{+}(X ; \mathscr{K}, \tau)$ denotes the set of measures in $\mathfrak{M}_{+}(X ; \mathscr{K})$ which are $\tau$-smooth w. r. t. $\mathscr{K}$.

In $\mathfrak{M}_{+}(X)$, two topologies, called the w-topology and the s-topology, are of interest to us.

The w-topology is defined as the weakest topology for which the mapping $\mu \rightarrow \mu X$ is continuous and all mappings $\mu \rightarrow \mu G$ are lower semicontinuous for every $G \in \mathscr{G}$. In other terms, if $\mu \in \mathfrak{M}_{+}(X)$ and if $\left(\mu_{\alpha}\right)$ is a net on $\mathfrak{M}_{+}(X)$, then $\mu_{\alpha}$ converges to $\mu$ in the w-topology, and we write $\mu_{\alpha} \stackrel{\stackrel{w}{\rightarrow}}{\rightarrow}$, if and only if $\mu_{\alpha} X \rightarrow \mu X$ and $\liminf \mu_{\alpha} G \geqslant \mu G \forall G \epsilon \mathscr{G}$.

The s-topology is defined as the weakest topology for which all mappings $\mu \rightarrow \mu A$, where $A \in \mathscr{B}$, are continuous. In other terms, if $\mu$ $\epsilon \mathfrak{M}_{+}(X)$ and if $\left(\mu_{\alpha}\right)$ is a net on $\mathfrak{M}_{+}(X)$, then $\mu_{\alpha}$ converges to $\mu$ in the s-topology, and we write $\mu_{\alpha} \stackrel{\mathrm{s}}{\rightarrow} \mu$ if and only if $\mu_{\alpha} A \rightarrow \mu A \forall A \in \mathscr{B}$.

The following five axioms are important for the investigations we are to carry out:
I. $\mathscr{K}$ is a $(\varnothing, \cup f, \cap c)$-paving.
II. $\mathscr{G}$ is a $(\varnothing, \cup f, \cap f)$-paving.
III. $K \backslash G \in \mathscr{K} \forall K \in \mathscr{K}, G \in \mathscr{G}$.
IV. $\mathscr{G}$ separate the sets in $\mathscr{K}$.
V. $\mathscr{K}$ is semicompact.

It is convenient to note the following easily established

LENarA. Assume that IV is satisfied. If $\mu_{\alpha} \stackrel{\mathrm{w}}{\rightarrow} \mu$ and if $\mu \epsilon \mathfrak{M}_{+}(X ; \mathscr{K})$, then we have

$\lim \sup \mu_{\alpha} K \leqslant \mu K \forall K \in \mathscr{K}$.

Assume now that $X$ is a topological space. Then we denote by $\mathscr{K}(X)$, $\mathscr{F}(X), \mathscr{G}(X)$, and $\mathscr{B}(X)$ the pavings on $X$ of compact sets, closed sets, open sets, and Borel sets, respectively. We always have $\mathscr{B}(\mathscr{K}(X)) \supseteq \mathscr{B}(X)$ and, in most cases, equality holds (if $X$ is a $k$-space, for instance if $X$ is locally compact or first countable, then equality holds). By $\mathfrak{M}_{+}(X)$ we denote the space of finite non-negative measures defined on $\mathscr{B}(X)$. The w-topology on $\mathfrak{M}_{+}(X)$ is defined via the paving $\mathscr{G}(X)$ and the s-topology on $\mathfrak{M}_{+}(X)$ is defined via the paving $\mathscr{B}(X)$. The w-topology on $\mathfrak{M}_{+}(X)$ is nothing but the familiar topology of weak convergence, which is well-known in, say Polish spaces (see [8]); in that case, and in others too, it is easy to prove that $\mu_{a}$ converges in the w-topology to $\mu$ if and only if $\mu_{a}(f) \rightarrow \mu(f)$ for every bounded continuous function $f$. 
$\mu \epsilon \mathfrak{M}_{+}(X)$ is regular if $\mu$ is regular w. r. t. $\mathscr{F}(X) . \mu \epsilon \mathfrak{M}_{+}(X)$ is tight if $\mu$ is regular w. r. t. $\mathscr{K}(X)$. The connection between tight measures and tight set-functions will be clear from the results of the next section. $\mu \epsilon \mathfrak{M}_{+}(X)$ is $\tau$-smooth if $\mu$ is $\tau$-smooth w.r.t. $\mathscr{F}(X)$. By $\mathfrak{M}_{+}(X ; r), \mathfrak{M}_{+}(X ; t)$ and $\mathfrak{M}_{+}(X ; \tau)$ we denote the spaces of regular, tight and $\tau$-smooth measures, respectively. A tight measure is regular and $\tau$-smooth. If $X$ is regular, then every $\tau$-smooth measure is regular. If $X$ is analytical (i.e. regular and continuous image of a Polish space), then every measure $\mu \epsilon \mathfrak{M}_{+}(X)$ is tight (see Hoffmann Jørgensen [6]). If $X$ is a $G_{\delta}$-space, then every measure is regular. If $X$ is regular, then $\mathfrak{M}_{+}(X ; \tau)$ in its w-topology is also regular. For further details and comments the reader is referred to Varadarajan [9] or to a forthcoming work by the author (Lecture notes in mathematics, vol. 133).

In the sequel, when we encounter pavings denoted by letters $\mathscr{K}, \mathscr{G}$ and $\mathscr{F}$, we shall always assume that sets denoted by the letters $K, G$ and $F$ are elements in $\mathscr{K}, \mathscr{G}$ and $\mathscr{F}$, respectively.

\section{Construction of measures.}

LEMMA 1. Let $X$ be an abstract set, $\mathscr{K}$ a $(\varnothing, \cup f, \cap \mathrm{f})$-paving on $X$ and $\lambda$ a finite set function on $\mathscr{K}$. Assume that $\lambda$ is tight. We then have:

(i) $\lambda$ is monotone and modular.

(ii) If $\lambda$ is $\sigma$-smooth at $\emptyset[\tau$-smooth at $\emptyset]$, then $\lambda$ is $\sigma$-smooth [ $\tau$-s mooth]

(iii) If the paving $\mathscr{K}$ is semi-compact [compact], then $\lambda$ is $\sigma$-smooth $[\tau-s m o o t h]$.

Proof. Olearly, $\lambda$ is monotone and $\lambda \varnothing=0$. For any subset $A$ of $X$ put $\mu A=\sup \{\lambda K \mid K \subseteq A\}$. Then $\mu$ is an extension of $\lambda$ and $\mu$ satisfies $\mu\left(K_{1} \backslash K_{2}\right)=\mu K_{1}-\mu K_{2} \forall K_{1} \supseteq K_{2}$. For any pair $K_{1}, K_{2}$ of sets in $\mathscr{K}$ we have $\mu\left(K_{1} \cup K_{2}\right)-\mu K_{2}=\mu\left(K_{1} \backslash K_{2}\right)=\mu K_{1}-\mu\left(K_{1} \cap K_{2}\right)$, thus $\lambda$ is modular. Assume now that $\lambda$ is $\sigma$-smooth at $\emptyset$ and let $\left\{K_{n}\right\}_{n \geqslant 1}$ be a decreasing sequence of sets in $\mathscr{K}$ such that $K_{0}=\bigcap_{1}^{\infty} K_{n} \in \mathscr{K}$. To a given $\varepsilon>0$ choose $K^{\prime} \subseteq K_{1} \backslash K_{0}$ such that $\lambda K^{\prime}+\lambda K_{0} \geqslant \lambda K_{1}-\varepsilon$. Then choose $n$ such that $\lambda\left(K_{n} \cap K^{\prime}\right) \leqslant \varepsilon$. Now $\lambda K_{n}=\lambda\left(K_{n} \cup K^{\prime}\right)+\lambda\left(K_{n} \cap K^{\prime}\right)-\lambda K^{\prime} \leqslant \lambda K_{1}+$ $+\varepsilon-\lambda K^{\prime}<\lambda K_{0}+2 \varepsilon$. This argument shows that $\lambda$ is $\sigma$-smooth. The $\tau$-smooth case is handled in the same way. The last assertion of the lemma follows easily from (ii). $\square$

THEOREM 1. Let $X$ be an abstract set, $\mathscr{K}$ a (Ø, $\cup \mathrm{f}, \cap \mathrm{c})$-paving on $X$ and $\lambda$ a tight content on $\mathscr{K}$ which is $\sigma$-smooth at $\emptyset$. Then $\lambda$ can be extended to a measure $\mu$ (not necessarily finite) such that $\mu$ is regular w.r.t. $\mathscr{K}$. Furthermore, we can achieve that any set in $\mathscr{B}(\mathscr{K})$ is measurable (i.e. is a member of the $\sigma$-field on which $\mu$ is defined).
Proof. For any subset $A$ of $X$ define $\mu A$ by $\mu A=\sup \{\lambda K \mid K \subseteq A\}$. $\mu$ is an extension of $\lambda, \mu$ is monotone and for disjoint sets $A$ and $B$ we have $\mu(A \cup B) \geqslant \mu A+\mu B$. Consider the class

$$
\mathscr{E}=\{E \mid \mu K=\mu(K \cap E)+\mu(K \backslash E) \forall K \in \mathscr{K}\} .
$$

$\mathscr{E}$ is closed under complementation and $\mathscr{E}$ contains every set $E$ such that $K \cap E \in \mathscr{K} \forall K \in \mathscr{K}$.

Let $\left\{E_{n}\right\}_{n \geqslant 1}$ be a sequence of sets in $\mathscr{E}$. Fix, for some time, $K \in \mathscr{K}$ and $\varepsilon>0$. Choose $K_{n}^{\prime} \subseteq K \cap E_{n}$ and $K_{n}^{\prime \prime} \subseteq K \backslash E_{n}$ such that

$$
\mu K \leqslant \mu K_{n}^{\prime}+\mu K_{n}^{\prime \prime}+\varepsilon 2^{-n}, \quad n \geqslant 1 .
$$

We claim that the inequality

$$
\lambda\left(\bigcup_{1}^{n} K_{i}^{\prime}\right)+\lambda\left(\bigcap_{s}^{n} K_{i}^{\prime \prime}\right) \geqslant \lambda K-\sum_{1}^{n} \varepsilon 2^{-i}
$$

holds for $n \geqslant 1$. (2) is true for $n=1$; therefore, in order to prove (2) for all $n \geqslant 1$, it is enough to establish the inequality

$$
\lambda\left(\bigcup_{1}^{n+1} K_{i}^{\prime}\right)+\lambda\left(\bigcap_{1}^{n+1} K_{i}^{\prime \prime}\right) \geqslant \lambda\left(\bigcup_{1}^{n} K_{i}^{\prime}\right)+\lambda\left(\bigcap_{1}^{n} K_{i}^{\prime \prime}\right)-\varepsilon 2^{-(n+1)}
$$

for $n \geqslant 1$. By lemma 1 , this inequality can be rewritten in the form

$$
\lambda K_{n+1}^{\prime}-\lambda\left(K_{n+1}^{\prime} \cap \bigcup_{1}^{n} K_{i}^{\prime}\right) \geqslant \lambda\left(K_{n+1}^{\prime \prime} \cup \bigcap_{1}^{n} K_{i}^{\prime \prime}\right)-\lambda K_{n+1}^{\prime \prime}-\varepsilon 2^{-(n+1)}
$$

and in this form the inequality follows from (1) and the fact that $K_{n+1}^{\prime} \cap$ $\cap \bigcup_{1}^{n} K_{i}^{\prime}$ and $K_{n+1}^{\prime \prime} \cup \bigcap_{1}^{n} K_{i}^{\prime \prime}$ are disjoint sets both contained in $K$.

By lemma $1, \lambda$ is $\sigma$-smooth and it follows from (2) that, for $n$ sufficiently large, the inequality

$$
\lambda\left(\bigcup_{1}^{n} K_{i}^{\prime}\right)+\lambda\left(\bigcap_{1}^{\infty} K_{i}^{\prime \prime}\right) \geqslant \lambda K-2 \varepsilon
$$

holds. On the one hand, it follows from (3) that

$$
\mu\left(K \cap \bigcup_{1}^{\infty} E_{i}\right)+\mu\left(K \backslash \bigcup_{1}^{\infty} E_{i}\right) \geqslant \lambda K-2 \varepsilon
$$

and, due to the freedom of choice of $\varepsilon$ and $K$, it follows from this that $\bigcup_{1}^{\infty} E_{i} \in \mathscr{E}$. On the other hand, it follows from (3) and the subadditivity of $\lambda$ that

we then see that

$$
\sum_{1}^{\infty} \lambda K_{i}^{\prime}+\lambda\left(\bigcap_{1}^{\infty} K_{i}^{\prime \prime}\right) \geqslant \lambda K-2 \varepsilon
$$

$$
\sum_{1}^{\infty} \mu E_{i}+\mu\left(K \backslash \bigcup_{1}^{\infty} E_{i}\right) \geqslant \lambda K
$$


holds for all $K \in \mathscr{K}$. Thus

$$
\mu\left(\bigcup_{1}^{\infty} E_{i}\right)=\sup \left\{\lambda K \mid K \subseteq \bigcup_{1}^{\infty} E_{i}\right\} \leqslant \sum_{1}^{\infty} \mu E_{i} .
$$

The restriction of $\mu$ to $\mathscr{E}$ has all the required properties. $\square$

Theorem 1 implies that a tight content defined on $\mathscr{K}(X)$ where $X$ is a topological space can be extended to a tight measure on $\mathscr{B}(X)$; this is the result proved by Kisyński (Theorem 1.2 of [7]).

It is instructive to observe that Theorem 1 also contains the following abstract extension theorem (Carathéodory's theorem):

Let $X$ be an abstract set, $\mathscr{A}$ a $(\cup \mathrm{f}, \mathrm{C})$-paving on $X$ (i.e. an algebra, and $\mu$ a finite, and additive set function defined on $\mathscr{A}$ such that $\mu$ is $\sigma$-smooth at $\varnothing$; then $\mu$ can be extended to a measure on $\sigma(\mathscr{A})$.

To see this, we just have to define $\mathscr{K}$ as the class of countable intersections of sets in $\mathscr{A}$ and to define $\lambda$ on $\mathscr{K}$ by $\lambda K=\inf \{\mu A \mid K$ $\subseteq A \in \mathscr{A}\}$.

We have also proved a result of the same type as Theorem 1 , but based on a class of functions instead of a class of sets; this result contains Daniell's extension theorem in much the same way as Theorem 1 contains Carathéodory's theorem. The proof is somewhat more technical than the proof of Theorem 1, and we shall not present it here

We shall now try to construct tight contents by "appioximation from outside".

LEMTIA 2. Assume that $(\bar{X}, \mathscr{K}, \mathscr{G})$ satisfies the axioms I, II and III and let $v$ denote a monotone, additive and subadditive set-function defined on $\mathscr{G}$ such that there to any $K \in \mathscr{K}$ exists a $G \in \mathscr{G}$ with $G \supseteq K$ and $\nu G<\infty$.

Define the set function $\lambda$ on $\mathscr{K}$ by $\lambda K=\inf \{\nu G \mid G \geq K\} ; K \in \mathscr{K}$. Then we have

(i) If $\lambda$ is additive, then $\lambda$ is a tight content.

(ii) If $\mathscr{G}$ separates the sets in $\mathscr{K}$, then $\lambda$ is additive.

(iii) If $v$ is $\sigma$-smooth at $\varnothing$ w.r.t. $\mathscr{K}[\tau$-smooth at $\varnothing$ w.r.t. $\mathscr{K}]$, then $\lambda$ is $\sigma$-smooth [ $\tau$-smooth].

Proof. $\lambda$ is finite, monotone and subadditive. Assume that $K_{1} \supseteq K_{2}$ and let $\varepsilon>0$ be given. Choose $G_{2} \supseteq K_{2}$ such that $\nu G_{2} \leqslant \lambda K_{2}+\varepsilon$. Put $K=K_{1} \backslash G_{2}$. Then $K \in \mathscr{K}$. If $G \supseteq K$, then $v G \geqslant v\left(G \cup G_{2}\right)-v G_{2} \geqslant \lambda K_{1}-v G$ $\geqslant \lambda K_{1}-\lambda K_{2}-\varepsilon$ and it follows that $\lambda K \geqslant \lambda K_{1}-\lambda K_{2}-\varepsilon$. What we have proved is that

$$
K_{1} \supseteq K_{2} \Rightarrow \sup \left\{\lambda K \mid K \subseteq K_{1} \backslash K_{2}\right\} \geqslant \lambda \dot{K}_{1}-\lambda K_{2} .
$$

(i) follows from this fact.

We omit the simple proof of (ii).
To prove (iii) assume that $v$ is, say $\tau$-smooth at $\emptyset$ w. r. t. $\mathscr{K}$. It follows that $\lambda$ is $\tau$-smooth at $\varnothing$. We shall prove from this that $\lambda$ is $\tau$-smooth. Let $\mathscr{K}^{*} \subseteq \mathscr{K}$ and assume that $\mathscr{K}^{*} \downarrow K_{0}$ and that $K_{0} \in \mathscr{K}$. Given $\varepsilon>0$, choose $G \supseteq K_{0}$ such that $\nu G \leqslant \lambda K_{0}+\varepsilon$. By considering the class $\left\{K^{*} \backslash G \mid K^{*} \in \mathscr{K}^{*}\right\}$ we see that $K^{*} \epsilon_{\mathscr{K}^{*}}$ ean be chosen so that $\lambda\left(K^{*} \backslash G\right) \leqslant \varepsilon$. From ( \pm ) it follows that $K \in \mathscr{K}$ can be chosen such that $K \subseteq K^{*} \cap G$ and such that $\lambda K \geqslant \lambda K^{*}-\lambda\left(K^{*} \backslash G\right)-\varepsilon$. It follows that $\lambda K^{*} \leqslant \lambda K+2 \varepsilon$ $\leqslant \nu G+2 \varepsilon \leqslant \lambda K_{0}+3 \varepsilon$. This argument shows that $\lambda$ is $\tau$-smooth. The $\sigma$-smooth case is handled analogously. $\square$

Remarks. We need not assume that $\mathscr{G}$ is closed under finite in tersections for the proof of (i) and (iii).

We need not assume that $v$ is additive for the proof of (i).

If $\mathscr{K}$ is a compact paving and if $\mathscr{G}$ separates points, then $\mathscr{G}$ separates the sets in $\mathscr{K}$.

If $y$ is modular, and if to any pair $K^{\prime}, K^{\prime \prime}$ of disjoint sets in $\mathscr{K}$ and to any positive $\varepsilon$ there exists a pair $G^{\prime}, G^{\prime \prime}$ of sets in $\mathscr{G}$ such that $G^{\prime} \supseteq K^{\prime}, G^{\prime \prime} \supseteq K^{\prime \prime}$ and such that $v\left(G^{\prime} \cap G^{\prime \prime}\right)<\varepsilon$, then $\lambda$ is additive.

THEOREM 2. Assume that $(X, \mathscr{K}, \mathscr{G})$ satisfies the axioms I-IV. Let $v$ be a monotone, additive and subadditive set function defined on $\mathscr{G}$ such that to any $K \in \mathscr{K}$ there exists a $G \epsilon \mathscr{G}$ with $G \supseteq K$ and $\nu G<\infty$. Lastly, assume that either $\mathscr{K}$ is a semicompact paving or else $v$ is $\sigma$-smooth at $\emptyset$ w. r.t. $\mathscr{K}$.

. Then there exists a largest measure $\mu$ regular w.r.t. $\mathscr{K}$ such that $\mu G$ $\leqslant \nu G \forall G \in \mathscr{G}$. This measure is given by the formula

$$
\mu A=\sup _{K} \inf _{\mathscr{K} \subseteq A} v, \quad A \in \mathscr{B}(\mathscr{K}) .
$$

If $v$ is $\tau$-smooth at $\varnothing$ w.r.t. $\mathscr{K}$, then the measure $\mu$ will be $\tau$-smooth w.r.t. $\mathscr{K}$.

This result follows in a straightforward manner from lemmas 1,2 and Theorem 1 and we leave the details of the proof to the reader.

In case $X$ is a topological space, we may apply Theorem 2 both to the case where $\mathscr{K}=\mathscr{K}(X), \mathscr{G}=\mathscr{G}(X)$ and to the case where $\mathscr{K}$ $=\left\{f^{-1}(0) \mid f\right.$ continuous and bounded $\}, \mathscr{G}=\{C K \mid K \in \mathscr{K}\}$.

In case $X$ is a normal topological space, $\mathscr{K}=\mathscr{F}(X)$, and $\mathscr{G}=\mathscr{G}(X)$, we obtain another special case of Theorem 2.

THEOREM 3. Let $X$ be a regular topological space and $v$ a finite, monotone and modular set function defined on $\mathscr{G}(X)$ such that $y$ is $\tau$-smooth at $\emptyset$ w.r.t. $\mathscr{F}(X)$.

Then there exists a largest measure $\mu$ in $\mathfrak{M}_{+}(X ; r)$ such that $\mu G$ $\leqslant \nu G \forall G \in \mathscr{G}(X)$. This measure is given by the formula

$$
\mu A=\sup _{F \subseteq A} \inf _{G \supseteq F} v G ; \quad A \in \mathscr{B}(X) .
$$

Furthermore, $\mu \in \mathfrak{M}_{+}(X ; \tau)$. 
Proof. Note that this result is contained in Theorem 2 in case $X$ is normal.

Define $\lambda$ on $\mathscr{F}(X)$ by $\lambda F=\inf \{\nu G \mid G \supseteq F\}$; By Lemma 2 and Theorem 1 we only have to prove that $\lambda$ is additive in order to see, that $\mu \in \mathfrak{D}_{+}(X ; \tau)$, where $\mu$ is defined by (6).

Let $F_{1}$ and $F_{2}$ be disjoint closed subsets of $X$ and let $\varepsilon>0$ be given. Consider the class consisting of all sets of the form $F_{1}^{\prime} \cap F_{2}^{\prime}$ where $F_{1}^{\prime}$ and $F_{2}^{\prime}$ are closed, and where the inclusions $F_{1}^{\prime} \supseteq G_{1} \supseteq F_{1}$ and $F_{2}^{\prime} \supseteq G_{2}$ $\supset F_{2}$ hold for some pair $G_{1}, G_{2}$ of open sets. Since $X$ is a regular space, we find that this class filters downward toward the empty set. By the assumption of $\tau$-smoothness we can thus find a set $F_{1}^{\prime} \cap F_{2}^{\prime}$ in our class and an open set $G$ containing $F_{1}^{\prime} \cap F_{2}^{\prime}$ such that $v(G)<\varepsilon$ holds. This implies that $\nu\left(G_{1} \cap G_{2}\right)<\varepsilon$ hold swhere $G_{1}$ and $G_{2}$ are open sets chosen such that $F_{1}^{\prime} \supseteq G_{1} \supseteq F_{1}$ and $F_{2}^{\prime} \supseteq G_{2} \supseteq F_{2}$ hold. According to the last of the remarks to Lemma 2 it follows that $\lambda$ is additive.

Having seen that $\mu \epsilon \mathfrak{M}_{+}(X ; \tau)$, it is easy to establish the first part of the theorem. $\square$

4. Compactness in the w-topology. We shall say that $\mathscr{A}_{1}$ dominates $\mathscr{A}_{2}$ and write $\mathscr{A}_{1}>\mathscr{A}_{2}$ if $\forall A_{2} \in \mathscr{A}_{2} \exists A_{1} \in \mathscr{A}_{1}: A_{1} \supseteq A_{2}$.

THEOREM 4. Assume that axioms I- $\nabla$ are satisfied and consider the space $\mathfrak{M}_{+}(X ; \mathscr{K})$ with the w-topology. Let $\left(\mu_{\alpha}\right)_{a \in D}$ be a net on $\mathfrak{M}_{+}(X ; \mathscr{K})$. Then $\left(\mu_{\alpha}\right)$ is compact if and only if the following two conditions hold:

(i) $\lim \sup \mu_{a} X<\infty$.

(ii) For every subclass $\mathscr{G}^{\prime}$ of $\mathscr{G}$ which dominates $\mathscr{K}$ we have

$$
\inf _{\mathscr{G}^{\prime \prime}} \limsup _{\alpha} \min _{G \in \mathscr{G}^{\prime \prime}} \mu_{\alpha}(\mathrm{C} G)=0,
$$

where the infimum is taken over all finite subclasses $\mathscr{G}^{\prime \prime}$ of $\mathscr{G}^{\prime}$.

Proof. Let us perhaps start to prove the more elementary part of the Theorem viz. the "only if" part. For this part of the proof we need not assume that the axioms I- $V$ hold. We assume that $\left(\mu_{\alpha}\right)$ is compact. Then clearly (i) must hold. If (ii) did not hold we would be able to find $\mathscr{G}^{\prime} \subseteq \mathscr{G}$ with $\mathscr{G}^{\prime}>\mathscr{K}$ and $\varepsilon>0$ such that to any pair $\left(\mathscr{G}^{\prime \prime}, \beta\right)$, where $\mathscr{G}^{\prime \prime}$ is a finite subfamily of $\mathscr{G}^{\prime}$ and $\beta \in D$ there exists an element $\alpha\left(\mathscr{G}^{\prime \prime}, \beta\right) \in D$ such that $\alpha\left(\mathscr{G}^{\prime \prime}, \beta\right) \geqslant \beta$ and such that

$$
\min _{G \in \mathscr{G}^{\prime \prime}} \mu_{a\left(\mathscr{G}^{\prime \prime}, \beta\right)}(\mathrm{C} G) \geqslant \varepsilon
$$

Consider the net $\left(\mu_{\alpha\left(\mathscr{G}^{\prime \prime}, \beta\right)}\right)_{\left(\mathscr{G}^{\prime \prime}, \beta\right)}$, where we use the natural ordering in the set of pairs $\left(\mathscr{G}^{\prime \prime}, \beta\right)$. This net is a subnet of $\left(\mu_{a}\right)$; therefore, it has a convergent subnet. Assume for simplicity that the net $\left(\mu_{\alpha\left(\mathscr{G}^{\prime \prime}, \beta\right)}\right)$ itself converges, and denote the limit measure by $\mu$. Then

$$
\begin{aligned}
\lim \mu_{a\left(\mathscr{G}^{\prime \prime}, \beta\right)} X & =\mu X=\sup _{K} \mu K \\
& \leqslant \sup _{K} \inf _{K \subseteq G_{0} \in \mathscr{G}^{\prime}} \mu G_{0} \\
& \leqslant \sup _{K} \inf _{K \subseteq G_{0} \mathscr{E}^{\prime}} \liminf \mu_{\alpha\left(\mathscr{G}^{\prime \prime}, \beta\right)} G_{0} \\
& =\sup _{K} \inf _{K \subseteq G_{0} \in \mathscr{G}^{\prime}}\left\{\lim \mu_{\alpha\left(\mathscr{G}^{\prime \prime}, \beta\right)} X-\limsup \mu_{a\left(\mathscr{G}^{\prime \prime}, \beta\right)}\left(C G_{0}\right)\right\} \\
& \leqslant \lim \mu_{a\left(\mathscr{G}^{\prime \prime}, \beta\right)} X-\varepsilon,
\end{aligned}
$$

a contradiction. Thus (ii) must hold.

Now assume that (i) and (ii) hold. Let $\left(\mu_{\alpha}\right)_{\beta \epsilon E}$ be a universal subnet of $\left(\mu_{a}\right)_{a \in D}$. Because of (i), $\lim \mu_{\alpha_{\beta}} A$ exists for all $A \in \mathscr{B}$ (and is finite). Let $\nu: \mathscr{G} \rightarrow R_{+}$be the set function $\nu G=\lim \mu_{\alpha_{\beta}} G ; G \in \mathscr{G}$. Then $\nu$ is monotone and modular. According to Theorem 2, $\mu: \mathscr{B} \rightarrow R_{+}$defined by

$$
\mu A=\sup _{K \subseteq A} \inf _{G \supseteq K} \nu G ; \quad A \epsilon \mathscr{B}
$$

is a measure in $\mathfrak{M}_{+}(X ; \mathscr{K})$ and $\mu \leqslant \nu$ on $\mathscr{G}$. To prove that $\mu_{\alpha_{\beta}} \stackrel{\mathrm{w}}{\rightarrow} \mu$ we need only prove that $\mu_{\alpha_{\beta}} X \rightarrow \mu X$. If this were not so we would be able to find $\varepsilon>0$ and a family $\left(G_{K}\right)_{K \in \mathscr{X}}$ of sets in $\mathscr{G}$ such that $G_{K}$ $\supseteq K \forall K \epsilon \mathscr{K}$ and such that, for every $K \epsilon \mathscr{K}, \mu_{a_{\beta}}\left(C G_{K}\right) \geqslant \varepsilon$, eventually. If we put $\mathscr{G}^{\prime}=\left\{G_{K} \mid K \in \mathscr{K}\right\}$, then $\mathscr{G}^{\prime}>\mathscr{K}$, and we find that, for every finite subfamily $\mathscr{G}^{\prime \prime}$ of $\mathscr{G}^{\prime}$ we have $\min \left\{\mu_{a_{\beta}}(C G) \mid G \in \mathscr{G}^{\prime \prime}\right\} \geqslant \varepsilon$, eventually. This is a contradiction. Thus $\mu_{\alpha_{\beta}} \stackrel{\text { }}{\rightarrow} \mu$. $\square$

COROLlaRY 1. Assume that axioms I-V are satisfied and let $\left(\mu_{\alpha}\right)_{a \in D}$ be a net on $\mathfrak{M}_{+}(X ; \mathscr{K})$ such that $\lim \sup \mu_{\alpha} X<\infty$.

(i) If the "tightness condition"

$$
\inf _{\mathbb{B} \in \mathscr{K}} \limsup _{\alpha} \mu_{\alpha}(\mathrm{C} K)=0
$$

holds, then $\left(\mu_{\alpha}\right)$ is compact.

(ii) If $\left(\mu_{\alpha}\right)$ is compact and if $\mathscr{K}$ is a compact paving, then

$$
\inf _{G \in G^{\prime}} \limsup _{\alpha} \mu_{\alpha}(C G)=0
$$

holds for every subfamily $\mathscr{G}^{\prime}$ of $\mathscr{G}$ with $\mathscr{G}^{\prime} \uparrow X$. (If we do not assume that $\mathscr{K}$ is compact, we obtain the same conclusion, but only for countable families $\mathscr{G}^{\prime}$ ).

The simple proof is left to the reader.

COROLLARY 2. Assume that axioms I- $\nabla$ are satisfied. Let $\mathscr{P}$ be a subset of $\mathfrak{M}_{+}(X ; \mathscr{K})$ and consider the following properties:
(i) $\mathscr{P}$ is net-compact
(ii) $\sup _{\mu \in \mathscr{P}} \mu X<\infty$, 


$$
\begin{aligned}
& \text { (iii) } \underset{\mathscr{G}^{\prime}>\mathscr{X}}{\forall} \inf _{\mathscr{G}^{\prime \prime} \subseteq \mathscr{G}^{\prime}} \sup _{\mu \in \mathbb{P}} \min _{G_{\in} \in \mathscr{G}^{\prime \prime}} \mu(\mathrm{C} G)=0 \text {, }
\end{aligned}
$$

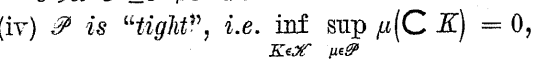

$$
\begin{aligned}
& \text { (v) } \underset{\mathscr{S}^{\prime} \uparrow X}{\forall} \inf _{G \in \mathscr{G}^{\prime}} \sup _{\mu \in \mathscr{P}} \mu(\mathrm{C} G)=0 .
\end{aligned}
$$

Then (i) is equivalent to (ii) and (iii), (ii) and (iv) implies (i), and, in case $\mathscr{K}$ is compact, (i) implies (v)..

Proof. Consider the net on $\mathfrak{M}_{+}(X ; \mathscr{K})$ defined by the identity map id: $\mathscr{P} \rightarrow \mathscr{P}$, where the domain of id is given the "diffuse" ordering $\left(\mu_{1} \leqslant \mu_{2}\right.$ for any pair of measures in $\left.\mathscr{P}\right)$. $\square$

As another corollary to Theorem 2 and the proof of Theorem 4 we obtain the result that $\mathfrak{M}_{+}(X ; \mathscr{K})$ in the w-topology is a Hausdorff space when I- $\nabla$ are satisfied (if $\left(\mu_{a}\right)$ is a convergent universal net: $\mu_{a} \stackrel{W}{\rightarrow} \sigma$, then $\left(\mu_{\alpha}\right)$ is compact and $\mu_{a}$ converges to the measure $\mu$ from the proof of Theorem 4. Clearly, $\sigma \leqslant \mu$ holds and, since $\sigma(X)=\mu(X)$, we must have $\sigma=\mu)$.

Remarks. If we in condition (iii) of Corollary 2 only take subclasses $\mathscr{G}^{\prime \prime}$ of $\mathscr{G}^{\prime}$ consisting of one set into consideration, then the condition we arrive at, in other words, the condition

$$
\underset{\mathscr{Y ^ { \prime }}>\mathscr{C}}{\forall} \inf _{G \in \mathscr{S}^{\prime}} \sup _{\mu \in \mathscr{P}} \mu(\mathrm{C} G)=0
$$

is equivalent to tightness of $\mathscr{P}$. This easily follows from the fact that $\mu \in \mathfrak{M}_{+}(X ; \mathscr{K}) \wedge K \in \mathscr{K} \Rightarrow \mu K=\inf \{\mu G \mid G \supseteq K\}$ (apply IV). In the case of Varadarajan's counterexample ([9], p. 225) one can prove that $\mathscr{P} \subseteq \mathfrak{M}_{+}(X ; \mathscr{K})$ is net-compact if and only if $\sup \{\mu X \mid \mu \in \mathscr{P}\}<\infty$ and

$$
\underset{\mathscr{S}^{\prime}>\mathscr{K} G_{1}, G_{2} \underline{G}^{\prime}}{\forall} \sup _{\mu \in \mathscr{P}} \min \left\{\mu\left(\mathrm{C} G_{1}\right), \mu\left(\mathrm{C} G_{2}\right)\right\}=0
$$

hold - and yet these conditions do not imply (7) (equivalent to tightness).

In ease $X$ is a locally compact topological space or a complete metric space (and $\mathscr{K}=\mathscr{K}(X), \mathscr{G}=\mathscr{G}(X)$ ), it is easy to see that net-compactness implies tightness (since, in these cases, $(v)$ implies tightness).

In Theorem 4 we need not assume that $\left(\mu_{a}\right)$ is a net on $\mathfrak{M}_{+}(X ; \mathscr{K})-$ -it is enough to consider a net on $\mathfrak{M}_{+}(X)$; then the conditions of the theorem are the necessary and sufficient conditions that every universal subnet of $\left(\mu_{\alpha}\right)$ converge to a measure in $\mathfrak{M}_{+}(X ; \mathscr{K})$.

We shall now derive some compactness results in case $\mathscr{K}$ is not assumed to be semicompact. We compensate for the lack of this axiom by assuming that $X \in \mathscr{K}$. In view of axioms II and III, this new assumption is equivalent to the requirement that $C G \in \mathscr{K} \forall G \in \mathscr{G}$.
THeorem 5. Assume that axioms I-IV are satisfied, that $X \in \mathscr{K}$, and that $x \notin K \Rightarrow \exists K^{\prime}: K^{\prime} \cap K=\varnothing \wedge x \in K^{\prime}$. Consider the space $\mathfrak{M}_{+}(X ; \mathscr{K}, \tau)$ with the w-topology and let $\left(\mu_{\alpha}\right)_{a \in D}$ be a net on $\mathfrak{M}_{+}(X ; \mathscr{K}, \tau)$. Then $\left(\mu_{a}\right)$ is compact if and only if $\lim \sup \mu_{a} X<\infty$ holds and, for every subclass $\mathscr{H}^{\prime}$ of $\mathscr{K}$ with $\mathscr{K}^{\prime} \downarrow \varnothing$, the relation

$$
\inf _{\mathbb{E}^{\prime} \in \mathscr{K}^{\prime}} \lim _{a} \sup _{a} \mu_{a} K=0
$$

holds.

Proof. Assume first that $\left(\mu_{a}\right)$ is compact. Then $\lim \sup \mu_{a} X<\infty$ holds. Assume, for the purpose of an indirect proof, that there exist $\mathscr{K}^{\prime} \downarrow \varnothing$ and $\varepsilon>0$ such that to any pair $(K, \beta)$ with $K \in \mathscr{K}^{\prime}$ and $\beta \in D$ there exists $\alpha(K, \beta) \in D$ with $\alpha(K, \beta) \geqslant \beta$ and $\mu_{\alpha(K, \beta)}(K) \geqslant \varepsilon$. Consider the net $\left(\mu_{\alpha(K, \beta)}\right)_{(K, \beta)}$, where the set of pairs $(K, \beta)$ is given the natural ordering. This net is a subnet of $\left(\mu_{\alpha}\right)$ and thus it has a convergent subnet. Assume for simplicity that $\left(\mu_{\alpha(K, \beta)}\right)$ itself converges, say $\mu_{a(K, \beta)} \stackrel{\underset{W}{\rightarrow}}{\rightarrow}$. Then we have

$$
0=\inf _{K_{0} \in \mathscr{K}^{\prime}} \mu K_{0} \geqslant \inf _{K_{0} \in \mathscr{E}^{\prime}} \lim \sup \mu_{a(\mathcal{K}, \beta)} K_{0} \geqslant \varepsilon,
$$

a contradiction. The "only if" part is now established.

Now assume that $\limsup \mu_{\alpha} X<\infty$ holds, and that (9) holds $\forall \mathscr{K}^{\prime} \downarrow \emptyset$. We need an auxillary result and consider a fixed class $\mathscr{K}^{\prime}$ with $\mathscr{K}^{\prime} \downarrow \varnothing$; define the class $\tilde{\mathscr{K}}$ as the class of those $\tilde{K} \in \mathscr{K}$ for which there exists a $G \in \mathscr{G}$ and a $K \in \mathscr{K}^{\prime}$ such that $\tilde{K} \supseteq G \supseteq K$. Due to IV and the added assumptions, we see that $\tilde{\mathscr{K}} \downarrow \emptyset$. To any $\varepsilon>0$ we can find $\tilde{K} \in \tilde{\mathscr{K}}$ such that $\lim \sup \mu_{a} \tilde{K} \leqslant \varepsilon$. Then we can also find $K \in \mathscr{K}^{\prime}$ and $G \epsilon \mathscr{G}$ with $G \supseteq K$ such that $\lim \sup \mu_{a} G \leqslant \varepsilon$. This argument proves the following:

$$
\underset{\mathscr{K}^{\prime} \downarrow}{\forall} \inf _{G \in \mathscr{G}^{\prime}} \lim \sup \mu_{a} G=0,
$$

where $\mathscr{G}^{\prime}$ is the class of those $G$ containing some set in $\mathscr{K}^{\prime}$.

Let $\left(\mu_{\alpha_{\beta}}\right)_{\beta \in E}$ be an universal subnet of $\left(\mu_{a}\right)_{a \in D}$. Define the set function $\nu$ on $\mathscr{G}$ by $\nu G=\lim \mu_{\alpha_{\beta}} G ; G_{\epsilon} \mathscr{G}$. Then $\nu$ is finite, monotone, and modular. Furthermore, it follows from (10) that $\nu$ is $\tau$-smooth at $\emptyset$ w.r.t. $\mathscr{K}$. Define $\mu$ by

$$
\mu A=\sup _{K \subseteq A} \inf _{G \supseteq K} Q ; \quad A \in \mathscr{B} .
$$

By Theorem $2, \mu \epsilon \mathfrak{M}_{+}(X ; \mathscr{K}, \tau)$ and $\mu \leqslant \nu$ on $\mathscr{G}$. Since $X \in \mathscr{K}$, we find that $\mu \bar{X}=v X=\lim \mu_{a_{\beta}} X$. Thus $\mu_{a_{\beta}} \stackrel{\mathbb{W}}{\rightarrow} \mu$. $\square$

As an easy consequence of Theorem 2 and the proof above we obtain the result that under the hypothesis of Theorem $\tilde{5}, \mathfrak{M}_{+}(X ; \mathscr{K}, \tau)$ is 
a Hausdorff space in the $\mathrm{w}$-topology. It can also be proved without much difficulty that $\mathfrak{M}_{+}(X ; \mathscr{K})$, and therefore also $\mathfrak{M}_{+}(X ; \mathscr{K}, \tau)$ is a regular space (for this we only need $C G \in \mathscr{K} \forall G \in \mathscr{G}$ and axiom IV). The relatively compact subsets of $\mathfrak{M}_{+}(X ; \mathscr{K}, \tau)$ are thus the same as the net-compact subsets, and by Theorem 5 we find that, under the hypothesis of that theorem, $\mathscr{P} \subseteq \mathfrak{M}_{+}(X ; \mathscr{K}, \tau)$ is relatively compact if and only if $\sup _{\mu \in \mathscr{P}} \mu X$ $<\infty$ and

$$
\underset{\mathscr{K}^{\prime} \downarrow \emptyset}{\forall \inf _{K \in \mathscr{X}^{\prime}}} \sup _{\mu \in \mathscr{P}} \mu K=0
$$

hold. This result has the interesting corollary that if $\mathscr{P}$ is relatively compact, then $c(\mathscr{P})$, the convex hull of $\mathscr{P}$, is relatively compact too. The corresponding result for the situation considered in Theorem 4 does not follow from that theorem and we wonder if it is true.

We shall now look into the case where $X$ is assumed to be a topological space. If $\mathscr{K}=\mathscr{K}(X)$ and $\mathscr{G}=\mathscr{G}(X)$, Theorem 4 and its corollaries apply directly. We can also apply Theorem 4 with $\mathscr{K}=\mathscr{K}(X)$ and $\mathscr{G}$ a $(\varnothing, \cup f, \cap f)$-paring of open sets that separate points. Note that if $\mathscr{G}$ is assumed to separate points and closed sets in the (rather weak) sense that $x \notin F \in \mathscr{F}(X) \Rightarrow \exists G \in \mathscr{G}: x \in G \wedge G \cap F=\emptyset$, then the $w$-topology in $\mathfrak{M}_{+}(X ; \mathscr{K}(X))$ based on $\mathscr{G}$ and the w-topology in $\mathfrak{M}_{+}(X ; \mathscr{K}(X))$ based on $\mathscr{G}(X)$ coincide. This remark gives rise to various criteria for weak convergence.

One application of Theorem 5 arises if $X$ is a topological space, $\mathscr{K}=\left\{f^{-1}(0) \mid f\right.$ continuous and bounded $\}, \mathscr{G}=\{C K \mid K K \in \mathscr{K}\}$ (see Theorem 25, p. 200 , of [9]).

The obvious application of Theorem 5 consists in taking as $X$ a normal topological space and $\mathscr{K}=\mathscr{F}(X), \mathscr{G}=\mathscr{G}(X)$. However, one can generalize the result thus obtained to a regular space. For this purpose we prove the following:

LEMMA 3. Let $X$ be a regular topological space and $\left(\mu_{\alpha}\right)_{a \in D}$ a net on $\mathfrak{M}_{+}(X)$. Then the following three conditions are equivalent:

$$
\begin{aligned}
& \text { (i) } \underset{\mathscr{F}^{\prime} \downarrow \emptyset}{\forall} \underset{F \in \mathscr{F}^{\prime}}{\inf } \lim \sup \mu_{\alpha} \stackrel{\circ}{F}=0, \\
& \text { (ii) } \underset{\mathscr{F}^{\prime} \downarrow \emptyset}{\forall} \underset{F \in \mathscr{F}^{\prime}}{\inf } \limsup _{a} \mu_{\alpha} F=0 \text {, } \\
& \text { (iii) } \underset{F^{\prime} \downarrow \emptyset \in \in G^{\prime}}{\forall} \inf \limsup \mu_{a} G=0 \text {, }
\end{aligned}
$$

where $\mathscr{G}^{\prime}=\left\{G \in \mathscr{G}(X) \mid G \supseteq F\right.$ for some $\left.F \in \mathscr{F}^{\prime}\right\}$.

Proof. Clearly, (iii) $\Rightarrow$ (ii) $\Rightarrow$ (i). Now assume that (i) holds and let $\mathscr{F}^{\prime} \subseteq \mathscr{F}(X)$ satisfy $\mathscr{F}^{\prime} \downarrow \varnothing$. Put $\mathscr{F}^{*}=\left\{F^{*} \in \mathscr{F}(X) \mid \stackrel{\circ}{F}^{*} \supseteq F\right.$ for some $\left.F \in \mathscr{F}^{\prime}\right\}$.
Since $X$ is regular, $\mathscr{F}^{*} \downarrow \varnothing$. Now

$$
\inf _{G \in \mathscr{G}^{\prime}} \lim \sup \mu_{a} G \leqslant \inf _{F \in \mathscr{F}^{*}} \lim \sup \mu_{a} \stackrel{\circ}{F^{\circ}}=0 .
$$

THeOREM 6. Let $X$ be a regular topological space and consider $\mathfrak{M}_{+}(X ; \tau)$ with the weak topology. Then a net $\left(\mu_{a}\right)_{\alpha \in D}$ on $\mathfrak{M}_{+}(X ; \tau)$ is compact if and only if limsup $\mu_{a} X<\infty$ holds and the condition of " $\tau$-smoothness",

$$
\inf _{F \in \mathscr{F}^{\prime}} \lim \sup \mu_{\alpha} F=0,
$$

holds for every class $\mathscr{F}^{\prime} \subseteq \mathscr{F}(X)$ with $\mathscr{F}^{\prime} \downarrow \emptyset$.

The proof is based on the same ideas as in the proof of Theorem 5 we only appeal to Lemma 3 and to Theorem 3 instead of Theorem 2.

The condition in Theorem 6 seems more manageable than the corresponding condition in the space $\mathfrak{M}_{+}(X ; t)$. Moreover, all nonpathological spaces, for instance all locally compact spaces, all complete metrizable spaces, and all analytical spaces are regular and satisfy $\mathfrak{M}_{+}(X ; \tau)=\mathfrak{M}_{+}(X ; t)$ so that Theorem 6 applies. It is easy to show that the conditions given in Theorem 6 need not be sufficient for compactness in the space $\mathfrak{M}_{+}(X ; t)$ if $\mathfrak{M}_{+}(X ; t)$ is not equal to $\mathfrak{M}_{+}(X, \tau)$. We can even give an example where $X$ is a separable metrizable space. The idea to construct this example is this: Let $X$ be a subset of $R$ with $\lambda_{*} X=0$ and $\lambda^{*} X=1$ and construct a sequence $\left(\mu_{n}\right)_{n \geqslant 1}$ of probability measures with finite support such that $\mu_{n} \stackrel{\text { w }}{\rightarrow} \lambda^{*}$. ( $\lambda=$ Lebesque measure).

\section{Compactness in the s-topology.}

Most of the results of this section have recently been obtained independantly and by different methods by P. Gänssler: „Compactness and sequential compactness in spaces of measures", to appear in Z. Wahrscheinlichkeitstheorie verw. Geb.

Theorem 7 (compare with Grothendieck [5], Théorème 2). Let $X$ be an abstract space and assume that axioms I-V are satisfied. Consider the space $\mathfrak{M}_{+}(X ; \mathscr{K})$ with the s-topology and let $\left(\mu_{a}\right)_{a \in D}$ be a net on $\mathfrak{M}_{+}(X ; \mathscr{K})$. with $\lim \sup \mu_{\alpha} X<\infty$. Then the following four conditions are equivalent:

(i) $\left(\mu_{a}\right)$ is compact; (ii) $\underset{K}{\forall} \inf \limsup \mu_{a}(G \backslash K)=0, \underset{A \in \mathscr{B}}{\forall} \inf _{K \subseteq A} \lim \sup \mu_{a}(A \backslash K)=0$;

(iii) $\underset{K}{\forall} \inf \lim \sup \mu_{a}(G \backslash K)=0, \inf _{K} \limsup \mu_{a}(C K)=0$;

(iv) $\forall \inf \lim \sup \mu_{a}(G \backslash K)=0,\left(\mu_{\alpha}\right)$ is compact in the w-topology.

Proof. (i) $\Rightarrow$ (ii): Both properties in (ii) are proved indirectly in a rather straightforward manner, and we leave the details to the reader.

Clearly, (ii) $\Rightarrow$ (iii) $\Rightarrow$ (iv). 
(iv) $\Rightarrow$ (i): Let $\left(\mu_{\alpha_{\beta}}\right)$ be a universal subnet of $\left(\mu_{\alpha}\right)$. Define: $\nu G$ $=\lim \mu_{\alpha_{\vec{\beta}}} G ; G \in \mathscr{G}$ and $\mu A=\sup _{K \subseteq A} \inf _{G \supseteq K} \nu G ; A \in \mathscr{B}$. Then $\mu \in \mathfrak{M}_{+}(X ; \mathscr{K})$. For any $K$ we have

$$
\mu K=\inf _{G \supseteq K} \lim \mu_{\alpha_{\beta}} G=\inf _{G \supseteq K}\left(\lim \mu_{\alpha_{\beta}} K+\lim \mu_{\alpha_{\beta}}(G \backslash K)\right)=\lim \mu_{\alpha_{\beta}} K .
$$

Then we have

$$
\mu A=\sup _{K \subseteq A} \mu K=\sup _{K \subseteq A} \lim \mu_{\alpha_{\beta}} K \leqslant \lim \mu_{\alpha_{\beta}} A
$$

for any $A \in \mathscr{B}$. Since $\left(\mu_{a}\right)$ is compact in the w-topology we also have $\mu X=\lim \mu_{\alpha_{\beta}} X$. Therefore, for any $A \in \mathscr{B}$, we find

$$
\mu A=\mu X-\mu(C A) \geqslant \lim \mu_{\alpha_{\beta}} X-\lim \mu_{\alpha_{\beta}}(C A)=\lim \mu_{\alpha_{\beta}} A .
$$

We now conclude that $\mu A=\lim \mu_{\alpha_{B}} A \forall A \in \mathscr{B} . \square$

Remark. If we also assume that $C K \in \mathscr{G} \forall K \in \mathscr{K}$, then (i) in Theorem 7 is equivalent to the condition

$$
\underset{G}{\forall} \inf _{K \subseteq G} \limsup \mu_{\alpha}(G \backslash K)=0 .
$$

Remark. If we change the assumptions of Theorem 7 to those of Theorem 5 and if we consider the space $\mathfrak{M}_{+}(X ; \mathscr{K}, \tau)$ with the s-topology, then the four conditions of Theorem 7 are still equivalent. The same remark applies if we change the assumptions to those of Theorem 6 and consider $\mathfrak{M}_{+}(X ; \tau)$ with the s-topology.

LEMMA 4. Assume again that axioms I- $\nabla$ hold, and consider the space $\mathfrak{M}_{+}(X ; \mathscr{K})$ with the s-topology. Then a subset $\mathscr{P} \subseteq \mathfrak{M}_{+}(X ; \mathscr{K})$ is relatively compact if and only if the following three conditions hold:

(i) $\sup _{\mu x} \mu \infty$.

(ii) $\mathscr{P}$ is net-compact in the w-topology.

(iii) For any sequence $\left(G_{n}\right)_{n \geqslant 1}$ of pairwise disjoint sets in $\mathscr{G}$ we have

$$
\lim _{n \rightarrow \infty} \sup _{\mu \in \mathscr{P}} \mu G_{n}=0 .
$$

Proof. First remark that we may speak of relative compactness instead of net-compactness since any space of measures is regular in the s-topology.

Assume now that $\mathscr{P}$ is relatively compact. Then (i) and (ii) hold If (iii) failed we would be able to find a sequence $\left(G_{n}\right)_{n \geqslant 1}$ of pairwise disjoint sets, a sequence $\left(\mu_{n}\right)_{n \geqslant 1}$ of measures in $\mathscr{P}$, and an $\varepsilon>0$ such that $\mu_{n} G_{n} \geqslant \varepsilon$ holds for all $n \geqslant 1$. Extract from $\left(\mu_{n}\right)$ a convergent subnet, say $\mu_{n_{\alpha}} \stackrel{\mathrm{s}}{\rightarrow} \mu$.
Since $\mu_{n_{\alpha}} G_{k} \rightarrow \mu G_{k} \forall k \geqslant 1$ and $\mu_{n_{\alpha}}\left(\bigcup G_{k}\right) \rightarrow \mu\left(\bigcup G_{k}\right)$, we find that

$$
\sum_{k=1}^{\infty}\left|\mu_{n_{\alpha}} G_{k}-\mu G_{k}\right| \rightarrow 0
$$

Choose $k_{0}$ such that $\mu G_{k}<\varepsilon / 2 \forall k \geqslant k_{0}$. Then choose $a$ such that $\sum_{1}^{\infty}\left|\mu_{n_{a}} G_{k}-\mu G_{k}\right|<\varepsilon / 2$ and such that $n_{a} \geqslant k_{0}$. We have

$$
\mu_{n_{\alpha}} G_{n_{\alpha}} \leqslant\left|\mu_{n_{\alpha}} G_{n_{\alpha}}-\mu G_{n_{\alpha}}\right|+\mu G_{n_{\alpha}} \leqslant \sum_{1}^{\infty}\left|\mu_{n_{\alpha}} G_{k}-\mu G_{k}\right|+\mu G_{n_{\alpha}}<\varepsilon,
$$

a contradiction. Thus (iii) must hold.

We shall now prove the more interesting part of the result and assume that (i), (ii) and (iii) hold. Due to the equiralence of (i) and (iv) in Theorem 7 , all we have to prove is that

$$
\underset{K}{\forall} \inf _{G \supseteq K \sup _{\mu \in \mathscr{P}}} \mu(G \backslash K)=0 .
$$
that

If this did not hold we would be able to find $K_{0} \in \mathscr{K}$ and $\varepsilon>0$ such

$$
\underset{G \supseteq K_{0}}{\forall} \underset{\mu \in \mathscr{P}}{\exists} \mu\left(G \backslash K_{0}\right)>\varepsilon .
$$

Consider any set $G^{\prime} \supseteq K_{0}$. Select $\mu \in \mathscr{P}$ such that $\mu\left(G^{\prime} \backslash K_{0}\right)>\varepsilon$. Choose $K \subseteq G^{\prime} \backslash K_{0}$ such that $\mu K>\varepsilon$. We can find $G^{\prime \prime} \supseteq K_{0}$ and $G \supseteq K$ such that $G^{\prime \prime} \cap G=\varnothing$. We can then repeat the argument using $G^{\prime \prime} \supseteq K_{0}$ as starting point. Since $\mathscr{K}<\mathscr{G}$ it is easy to start the process; we can now see that there exist a decreasing sequence $\left(G_{n}^{\prime}\right)_{n \geqslant 1}$ with $G_{n}^{\prime} \supseteq K_{0} \forall n$ and a sequence $\left(\mu_{n}\right)_{n \geqslant 1}$ of measures in $\mathscr{P}$, and a sequence $\left(G_{n}\right)_{n \geqslant 1}$ such that $G_{n} \subseteq G_{n}^{\prime} \backslash G_{n+1}^{\prime}$ and $\mu_{n} G_{n}>\varepsilon ; n \geqslant 1$. Clearly, this contradicts condition (iii).

THEOREM 8. (compare with Grothendieck [5], Théorème 2). Assume that axioms I-V hold, and that, furthermore, the condition

$$
K \subseteq G \Rightarrow \exists G^{\prime}, G^{\prime \prime}: K \subseteq G^{\prime} \subseteq \mathrm{C} G^{\prime \prime} \subseteq G
$$

is satisfied.

Consider the space $\mathfrak{M}_{+}(X ; \mathscr{K})$ with the s-topology. Then a subset $\mathscr{P}$ of $\mathfrak{M}_{+}(X ; \mathscr{K})$ is relatively compact if and only if the following two conditions are satisfied:

(i) $\sup _{\mu \in \mathscr{P}^{P}} \mu X<\infty$.

(ii) For every sequence $\left(G_{n}\right)_{n \geqslant 1}$ of pairwise disjoint sets in $\mathscr{G}$ we have

$$
\lim _{n \rightarrow \infty} \sup _{\mu \in \mathscr{P}} \mu G_{n}=0 \text {. }
$$

Proof. In view of Lemma 4 we need only prove that (i) and (ii) imply that $\mathscr{P}$ is net-compact in the w-topology. We prove this by proving Studia Mathematica XXXVI.3 
that $\mathscr{P}$ is "tight". If this were not so, we could find $\varepsilon>0$ such that $\forall K \exists \mu \in \mathscr{P}: \mu(\mathrm{C} \dot{K})>\varepsilon$. Choose a sequence $\left(\varepsilon_{n}\right)_{n \geqslant 1}$ of positive numbers such that $\sum_{1}^{\infty} \varepsilon_{n} \leqslant \varepsilon / 2$.

We claim that we can construct a sequence $\left(K_{n}\right)_{n \geqslant 0}$ of sets in $\mathscr{K}$, two sequences $\left(G_{n}^{\prime}\right)_{n \geqslant 1}$ and $\left(G_{n}^{\prime \prime}\right)_{n \geqslant 1}$ of sets in $\mathscr{G}$, and a sequence $\left(\mu_{n}\right)_{n \geqslant 1}$ of measures in $\mathscr{P}$ such that (a)-(f) below hold:

(a) the $K_{n}$ 's are pairwise disjoint,

(b) $\mu_{n} K_{n} \geqslant \varepsilon \forall n \geqslant 1$

(c) $G_{n}^{\prime} \supseteq K_{n} \forall n \geqslant 1$,

(d) $G_{n}^{\prime \prime} \supseteq \bigcup^{n-1} K_{i} \forall n \geqslant 1$,

(e) $G_{n}^{\prime} \cap G_{n}^{\prime \prime}=\emptyset \forall n \geqslant 1$,

(f) $\sup _{\mu \in \mathscr{P}} \mu\left(\mathrm{C}\left(G_{n}^{\prime \prime} \cup K_{n}\right)\right) \leqslant \varepsilon_{n} \forall n \geqslant 1$.

We start the construction by taking as $K_{0}$ any set in $\mathscr{K}\left(K_{0}=\emptyset\right.$ will do).

Assume now, for some $n \geqslant 0$, that $K_{i}$ for $0 \leqslant i \leqslant n$, that $G_{i}^{\prime}$ for $1 \leqslant i \leqslant n$, that $G_{i}^{\prime \prime}$ for $1 \leqslant i \leqslant n$, and that $\mu_{i}$ for $1 \leqslant i \leqslant n$ are constructed so that the relevant parts of (a)-(f) are satisfied. Since $\bigcup_{0}^{n} K_{i} \in \mathscr{K}$, we can find $\mu_{n+1} \epsilon \mathscr{P}$ with $\mu_{n+1}\left(\mathrm{C}\left(\bigcup_{0}^{n} K_{i}\right)\right)>\varepsilon$. Choose $K_{n+1} \subseteq \mathrm{C}\left(\bigcup_{0}^{n} K_{i}\right)$ such that $\mu_{n+1} K_{n+1} \geqslant \varepsilon$. By what was proved in Lemma 4, we can find $\tilde{G}_{n+1}$ $\supseteq K_{n+1}$ such that $\sup \left\{\mu\left(\tilde{G}_{n+1} \backslash K_{n+1}\right) \mid \mu \epsilon \mathscr{P}\right\} \leqslant \varepsilon_{n+1}$. By axiom IV we may and do assume that $\tilde{G}_{n+1} \cap \bigcup_{0}^{n} K_{i}=\varnothing$. Choose, according to (11) $G_{n+1}^{\prime}$ and $G_{n+1}^{\prime \prime}$ such that

$$
K_{n+1} \subseteq G_{n+1}^{\prime} \subseteq \mathrm{C} G_{n+1}^{\prime \prime} \subseteq \tilde{G}_{n+1} .
$$

We see that the relevant parts of (a)-(f) continue to hold when we take $K_{n+1}, \mu_{n+1}, G_{n+1}^{\prime}$ and $G_{n+1}^{\prime \prime}$ into consideration. By induction we thus establish (a)-(f).

Define the sequence $\left(G_{n}\right)_{n \geqslant 1}$ by $G_{1}=G_{1}^{\prime}, G_{n}=G_{n}^{\prime} \cap \bigcap_{1}^{n-1} G_{i}^{\prime \prime} ; n \geqslant 2$. For $n<m$ we find that $G_{n} \cap G_{m} \subseteq G_{n}^{\prime} \cap G_{n}^{\prime \prime}=\varnothing$ so that the $G_{n}{ }^{2} \mathrm{~s}$ are pairwise disjoint. We now cary out the following calculations, noting that $G_{n}^{\prime} \subseteq \mathrm{C}\left(\bigcup_{0}^{n-1} K_{i}\right)$ :

$$
\mu_{n} G_{n}=\mu_{n} G_{n}^{\prime}-\mu_{n}\left(G_{n}^{\prime} \backslash \bigcap_{1}^{n-1} G_{i}^{\prime \prime}\right)
$$

$$
\begin{aligned}
& \geqslant \mu_{n} K_{n}-\mu_{n}\left(\bigcup_{1}^{n-1}\left(\mathrm{C} G_{i}^{\prime \prime} \cap G_{n}^{\prime}\right)\right) \\
& \geqslant \varepsilon-\mu_{n}\left(\bigcup_{1}^{n-1} \mathrm{C}\left(G_{i}^{\prime \prime} \cup K_{i}\right)\right) \\
& \geqslant \varepsilon-\sum_{1}^{\infty} \mu_{n}\left(\mathrm{C}\left(G_{i}^{\prime \prime} \cup K_{i}\right)\right) \\
& \geqslant \varepsilon-\sum_{1}^{\infty} \varepsilon_{n} \geqslant \varepsilon / 2 .
\end{aligned}
$$

Since $\mu_{n} G_{n} \geqslant \varepsilon / 2 \quad \forall n \geqslant 1$ we arrive at a contradiction with condition (ii). $\square$

A similar remark as the second remark to Theorem 7 applies to Theorem 8.

Corollakr 3 (compare with Dieudonné [2], Proposition 8). Assume that axioms I-V are satisfied, that $\mathscr{G}$ is closed under countable unions, and that condition (11) from Theorem 8 holds. Let $\left(\mu_{n}\right)_{n \geqslant 1}$ be a sequence on $\mathfrak{M}_{+}(X ; \mathscr{K})$ and assume that $\lim \mu_{n} G$ exists for every $G \in \mathscr{G}$. Then there exists a measure $\mu \in \mathfrak{M}_{+}(X ; \mathscr{K})$ such that $\mu_{n} \stackrel{\mathrm{s}}{\rightarrow} \mu$.

Proof. Due to (11), $X_{\epsilon} \mathscr{G}$ and we see that (i) Theorem 8 holds. To establish (ii), Theorem 8 is essentially equivalent to proving that the result holds in case $X$ is countable and $\mathscr{G}=D(X)$; the main fact needed in order to prove this is contained in a result of Nikodym (see III, 7.4 of [3]). Having seen that $\left(\mu_{n}\right)_{n \geqslant 1}$ is compact, it is easy to see that $\left(\mu_{n}\right)$ converges since any two limit measures agree on the sets in $\mathscr{G}$ - and therefore on all sets in $\mathscr{B}$. $\square$

The idea to prove this corollary by establishing a result like Theorem 8 is due to Grothendieck.

We shall leave it to the reader to see what comes out of the results in this section in case $X$ is a topological space.

Remark. Throughout this section we have worked with non-negative measures and not, as is perhaps. more proper, with signed measures. To indicate briefly how the general case can be handled, we mention the following result: Assume that axioms I- $\nabla$ are fulfilled and let $\mathscr{P} \subseteq$ $\mathfrak{M}(X, \mathscr{K})$ be given satisfying $\sup \{|\mu|(X) \mid \mu \epsilon \mathscr{P}\}<\infty$ (that we may assume this, is well-known allthough not entirely trivial). Then the following conditions are all equivalent:

(i) $\mathscr{P}$ is relatively compact (in the s-topology);

(ii) for every sequence $\left(E_{n}\right)_{n \geqslant 1}$ of pairwise disjoint sets in $\mathscr{B}$ we have

$$
\varliminf_{n \rightarrow \infty} \sup _{\mu \in \mathscr{P}}|\mu|\left(E_{n}\right)=0 \text {; }
$$


(iii) $\forall K \inf _{G \supseteq K} \sup _{\mu \in \mathscr{P}}|\mu|(G \backslash K)=0$ and $\forall A \in \mathscr{B} \inf _{K \subseteq A} \sup _{\mu \in \mathscr{P}}|\mu|(A \backslash K)=0$.

To prove this, one proves the implications (i) $\Rightarrow$ (ii) $\Rightarrow$ (iii) $\Rightarrow$ (i). The ideas involved in the proof are either contained in this section or else well-known from other sources.

I do not know if the compactness results for nets on $\mathfrak{M}_{+}(\ldots)$ generalize to nets on $\mathfrak{M}(. .$.$) .$

\section{References}

[1] N. Bourbaki, Eléments de mathématique, Livre III, Topologie générale, 2nd ed., Paris 1951.

[2] J. Dieudonné, Sur la convergence des suites de mesures de Radon, Anais Acad. Brasil. Ci. 23 (1951), p. 21-38 and p. 277-282.

[3] N. Dunford and J. T. Schwartz, Linear operators, Part 1, General theory, New York 1958

[4] X. Fernique, Processus linéaires, processus généralisés, Ann. Inst. Fourier, Grenoble, 17 (1967), p. 1-92.

[5] A. Grothendieck, Sur les applications linéaires faiblement compactes d'espaces du type $O(K)$, Canadian J. Math. 5 (1953), p. 129-173.

[6] J. Hoffman Jørgensen, The theory of analytic spaces, Aarhus: Various publications series, 1970 .

[7] J. Kisyński, On the generation of tight measures, Studia Math. 30 (1968), p. $141-151$

[8] Yu. V. Prohorov, Convergence of random processes and limit theorems in probability theory, Theor. Probab. Appl. 1 (1956), p. 157-214.

[9] V. S. Varadarajan, Measures on topological spaces, Amer. Math. Soc. Transl., ser. II, 48 (1965), p. 161-228.

THE UNIVERSITY OF COPENHAGHN

Reçu par la Rédaction le 12. 6. 1969

\section{On isometries of normed linear spaces}

by

\section{KOEHLER (Oxford) and PETER ROSENTHAL* (Toronto)}

1. Introduction. Fixman [2] showed that it was not possible to develop a general spectral theory for invertible isometries on an arbitrary Banach space. The purpose of this note is to obtain a simple characterization of the isometries on a normed linear space (real or complex), in terms of semi-inner products. This is then used to show that eigenvectors corresponding to distinct eigenvalues of an isometry are "orthogonal", and to establish some facts about the point spectrum of isometries. In some minor respects, invertible isometries have spectral behavior like unitary operators on Hilbert space. Also, conditions are found that are necessary and sufficient for a given operator on a normed linear space to be equivalent to an isometry on an equivalent normed linear space.

Lumer [8] has shown that in any normed linear space $X$, one can construct a semi-inner-product $[\cdot, \cdot]$ (there may be more than one), i.e., a mapping from $X \times X$ into $O$ such that

(i) $[x, x]=\|x\|^{2}$,

(ii) $[a x+b y, z]=a[x, z]+b[y, z]$,

(iii) $|[x, y]|^{2} \leqslant[x, x] \cdot[y, y]$.

Giles has shown ([3], p. 437 ) that it is always possible to choose a semi-inner-product such that

(iv) $[x, a y]=\bar{a}[x, y]$.

We shall assume for the rest of the paper that all semi-inner-products satisfy (iv).

We shall follow James [6] in saying that $x$ is orthogonal to $y$ if $\|x\|$ $\leqslant\|x+a y\|$ for all scalars $a$.

* Part of the research by the first author was performed at the U.S.A.F. Aerospace Research Laboratories while in the capacity of an Ohio State University Research Foundation Visiting Research Associate under Contract F 3361567 C 1758

The research of the second author was partially supported by a grant from the National Research Council of Canada. 